\title{
Dynamical Brittle Fractures of Nanocrystalline Silicon using Large-Scale Electronic Structure Calculations
}

\author{
Takeo Hoshi and Takeo Fujiwara \\ Department of Applied Physics, University of Tokyo, \\ Bunkyo-ku, Tokyo 113-8656, Japan
}

\begin{abstract}
A hybrid scheme between large-scale electronic structure calculations is developed and applied to nanocrystalline silicon with more than $10^{5}$ atoms. Dynamical fracture processes are simulated under external loads in the [001] direction. We shows that the fracture propagates anisotropically on the (001) plane and reconstructed surfaces appear with asymmetric dimers. Step structures are formed in larger systems, which is understood as the beginning of a crossover between nanoscale and macroscale samples.
\end{abstract}


Silicon is one of ideally brittle materials and its fracture behavior is studied intensively, because we can obtain essentially dislocation-free single crystals. A pioneering theory of brittle fracture was given, in 1920's, by Griffith ${ }^{\underline{1}}$ within a continuum theory, which is the foundation of the present understanding of brittle fractures ${ }^{2}$. The fracture in single crystals should be investigated also in atomistic pictures, on the point of how and why the fracture path is formed and propagates in the crystalline geometry. This point includes surface reconstruction processes. Since fracture is a thermal non-equilibrium process, the atomic structure on a cleavage surface can be different from that on equilibrium clean surfaces. For example, the easiest cleavage plane in macroscale samples of silicon is the (111) plane, in which the surface structure is not the ground state $(7 \times 7)$ structure but a metastable $2 \times 1$ structure ${ }^{3,4}$.

This letter is devoted to the atomistic fracture behaviors in nanocrystalline silicon, especially, its possible difference from macroscale samples. Such a difference can be expected, as explained below; Now a typical atomistic length in silicon is defined as $d_{0} \equiv \sqrt[3]{v_{0}} \approx 3$ $\AA$, where $v_{0}$ gives the volume per atom. The essence of the Griffith theor $\underline{y}^{\underline{1}}$ is the energy competition between the energy gain of the strain relaxation and the loss of the surface formation energy. The former energy is a volume term proportional to (length) ${ }^{3}$, while the latter energy is a surface term proportional to (length) ${ }^{2}$. As analogous to the theory of nucleation ${ }^{5}$, the dimensional analysis gives the critical crack length for the spontaneous fracture propagation. The critical crack length $c_{\mathrm{G}}$ is given as ${ }^{1.2}$

$$
c_{G} \approx \frac{\gamma E}{\sigma^{2}}
$$

with the stress $\sigma$, the Young modulus $E\left(\approx 10^{2} \mathrm{GPa}\right)$ and the surface energy per unit area $\gamma$. The value of $\gamma$ was estimated to be in the order of $1 \mathrm{~J} / \mathrm{m}^{26.7}$, which can be reduced to the bond breaking energy $\left(\gamma d_{0}^{2} \approx 1 \mathrm{eV}\right)$ in the atomistic picture. In a recent experiment with macroscale samples ${ }^{8}$, the stress is $\sigma \approx 10^{1} \mathrm{MPa}$ and Eq. (11) gives a macroscale length $\left(c_{G} \approx 1 \mathrm{~mm}\right)$. Since the length $c_{\mathrm{G}}$ is not dependent on the sample size $L$, the fracture behavior can be expected to be different from the above picture, in case that the sample size $L$ is smaller than the critical length $c_{\mathrm{G}}\left(L<c_{\mathrm{G}}\right)$. In this letter, we will see such a situation in nanocrystalline silicon, in which the numbers of atomic layers for these lengths $\left(\approx c_{\mathrm{G}} / d_{0}, L / d_{0}\right)$ are not macroscale numbers.

For atomistic fracture simulations of silicon crystals, a recent work of classical modelings 9 


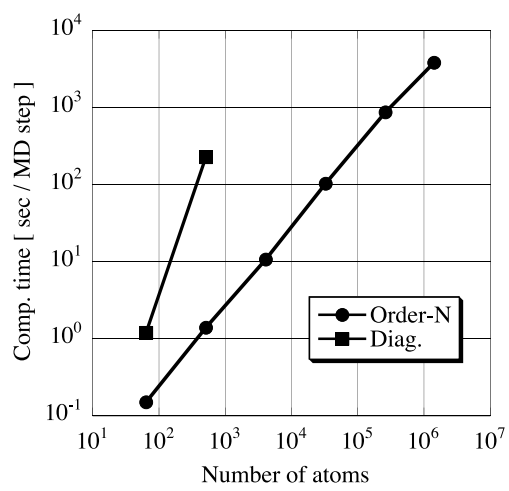

FIG. 1: The computational time for bulk silicon as the function of the number of atoms, up to 1,423,909 atoms; The CPU time is measured for one time step in the molecular dynamics (MD) simulation. A tight-binding Hamiltonian is solved using the perturbative order-N method and the exact diagonalization. We use a standard workstation with single CPU and 2 GB RAMs.

was done with $10^{5}$ atoms. A more recent work ${ }^{7}$, however, pointed out the limited applicability of classical modelings and the importance of electronic structure calculations. On the other hand, there are several ab initio calculations with $10^{2}$ atoms $\frac{6.7}{}$. Due to the system size of simulations, these investigations are limited in situations, such as the preparation of the initial cleavage plane in which the reconstructed surface structure is assumed. Therefore, large-scale electronic structure calculations are essential.

So far, we have developed several order- $\mathrm{N}$ methods for large-scale electronic structure calculations ${ }^{10}$. The order- $\mathrm{N}$ method is the general name of methods in which the computational cost is proportional to the system size $(N)$. We have developed the variational and perturbative order- $\mathrm{N}$ methods based on generalized Wannier states ${ }^{10,11}$. The Wannier states $\left\{\phi_{i}\right\}$ are localized and the index $i$ denotes its localization center. The equation for wave functions is common between the two methods and is given by the one-body density $\operatorname{matrix}\left(\rho \equiv \sum_{i}^{o c c .}\left|\phi_{i}\right\rangle\left\langle\phi_{i}\right|\right)^{10}$. In the computational algorithm, the perturbative method is simpler than the variational method. Figure 1 demonstrates our large-scale calculation among $10^{2}-10^{6}$ atoms.

With the above two methods, we now construct a novel hybrid scheme, in which the variational method is used only for the wave functions whose centers locate near fracture regions. The regions contain, typically, $4 \times 10^{4}$ electrons. Some of such wave functions change their character dynamically from the bulk ( $\mathrm{sp}^{3}$ bonding) states to surface ones, as 
discussed later. The other wave functions, in bulk regions, keep the character of the bulk bonding states and can be obtained by the perturbative method. The wave functions $\left\{\phi_{i}\right\}$ calculated by the two methods are commonly used for constructing the one-body density matrix $\rho$. Any physical quantity is expressed by the density matrix ${ }^{10}$ and is well defined in the present hybrid scheme.

The present work is based on a transferable tight-binding Hamiltonian with $\mathrm{s}$ and $\mathrm{p}$ orbitals 12 . It is used for several crystalline phases and non-crystalline phases, such as liquid 12 and surfaces 13 . Since the fracture is the formation of surfaces in a bulk region, the theory should reproduce the atomic structures both in bulk and surface phases, which is satisfied in the present Hamiltonian. The essence of the quantum mechanical freedoms is the fact that the $\mathrm{sp}^{3}$-hybridized bonds are formed in the bulk region, while are not on surfaces. To analyze the hybridization freedom, a parameter $f_{\mathrm{s}}^{(j)}$ is defined, for a wave function $\phi_{j}$, as

$$
f_{\mathrm{s}}^{(j)} \equiv \sum_{I}\left|\left\langle\phi_{j} \mid I s\right\rangle\right|^{2}
$$

where $|I s\rangle$ is the s orbital at the $I$-th atom. For example, $f_{\mathrm{s}}=1 / 4$ in an ideal $\mathrm{sp}^{3}$ hybridized state.

In this letter, we focus on the $\mathrm{Si}(001)$ surface, a standard template of the modern silicon technology. A characteristic feature in the $\mathrm{Si}(001)$ surface is the formation of asymmetric dimers 14.15 . The asymmetric dimer is connected by a ' $\sigma$ ' bonding state. Another state is localized on the 'up' atom, the dimerized atom near the vacuum region. This localized state is called ' $\pi$ ' state, because the direction of its $\mathrm{p}$ components is nearly perpendicular to the dimer bond. Here an energy quantity is defined as

$$
\Delta \varepsilon_{i}^{(\mathrm{cov})} \equiv\left\langle\phi_{i}|H| \phi_{i}\right\rangle-\left[f_{\mathrm{s}}^{(i)} \varepsilon_{\mathrm{s}}+\left(1-f_{\mathrm{s}}^{(i)}\right) \varepsilon_{\mathrm{p}}\right]
$$

A negative value of $\Delta \varepsilon_{i}^{(\mathrm{cov})}$ corresponds to the energy gain of a covalent bonding. The ' $\sigma$ ' state has the gain of $\Delta \varepsilon_{i}^{(\operatorname{cov})} \approx-2 \mathrm{eV}$, which mainly contributes to the dimerization energy (about $-2 \mathrm{eV})^{15}$. The ' $\pi$ ' state has much smaller $\Delta \varepsilon_{i}^{(\mathrm{cov})}$, which is comparable to the energy difference between the asymmetric and symmetric dimers (the order of $0.1 \mathrm{eV})^{15}$.

The simulation details are as follows; The hybrid order- $\mathrm{N}$ scheme is used for systems with $10^{4}$ atoms or more. In systems smaller than the above size, the variational method is used in the whole region. The samples are isolated tetragonal clusters, whose geometries are labeled with the number of atomic layers, such as $n_{100} \times n_{010} \times n_{001}$ or $n_{110} \times n_{1 \overline{1} 0} \times n_{001}$. As 
the boundary condition, the Wannier states at the sample surfaces are terminated by fixed $\mathrm{sp}^{3}$ bonding states and are not reconstructed. The time step of the molecular dynamics is 3 fs. The total kinetic energy is controlled to be that with $300 \mathrm{~K}$ by the Nose thermostat method $^{16}$. The numerical accuracy is checked among bulk, surface and fracture properties, such as the elastic constants, the dimer formations on the clean (001) surface, and the critical stress for fracture. The last quantity is checked, with smaller samples, in comparison with the standard diagonalization. The calculated fracture propagation velocity is always in the same order of, but less than, the Rayleigh surface wave velocity $(4.5 \mathrm{~km} / \mathrm{s})$, as expected from the continuum theory ${ }^{2}$.

For fracture propagations, external loads in the [001] direction are imposed. During the simulations, the external loads can be dynamically controlled by the atoms on the sample surfaces in the $z$ direction. These atoms are fixed or under artificial constantvelocity motions in the $z$ axis. The velocity, typically $10^{-2} \mathrm{~km} / \mathrm{s}$, is much smaller than that of observed fracture propagation velocities $(\mathrm{km} / \mathrm{s})$. As a seed of fractures, a short range repulsive potential is imposed on one particular pair of atoms, as a defect bond. For smaller samples, the simulations begin without initial deformations. The fracture always occurs with the external loads in the order of $\sigma \approx 1 \mathrm{GPa}$, which corresponds to the strain energy of $\sigma d_{0}^{3} \approx 0.1 \mathrm{eV}$ per atom. For larger samples, the simulations begin with initial static deformations in the above magnitude of external loads. The length $c_{\mathrm{G}}$ in Eq. (11) is calculated as $c_{G} \approx 100 \mathrm{~nm}$, which is longer than the present sample sizes $(L \leq 20 \mathrm{~nm})$.

In results, a two-stage reconstruction process is commonly observed as the elementary process during successive bond breakings; In Fig. 2(a), we monitor the one-electron energy

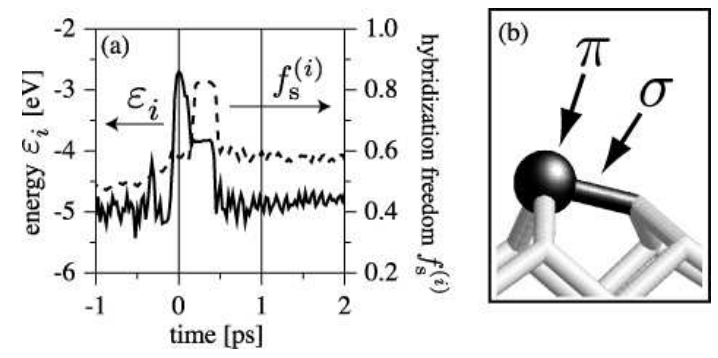

FIG. 2: (a) Elementary reconstruction process with the one-electron energy $\varepsilon_{i}$ and the weight on s orbitals $f_{\mathrm{s}}^{(i)}$. (b) An asymmetric dimer on a resultant crack. The black rod and black ball correspond to the ' $\sigma$ ' and ' $\pi$ ' states, respectively. 


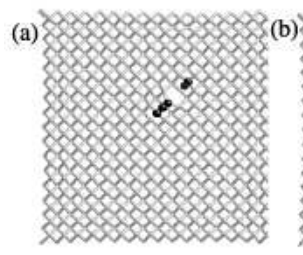

(d)

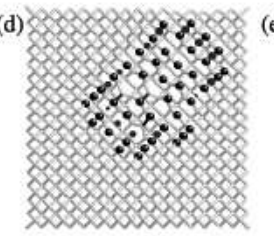

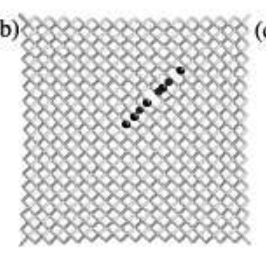
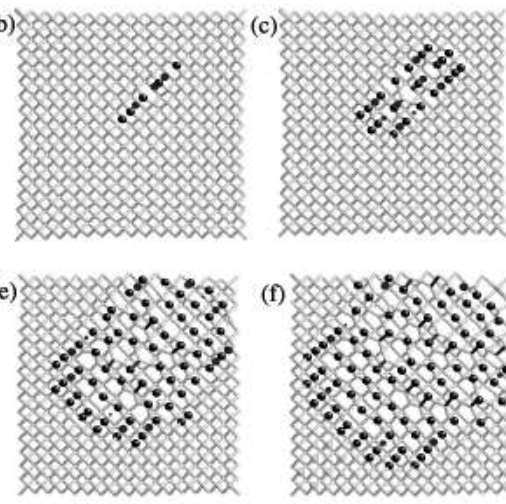

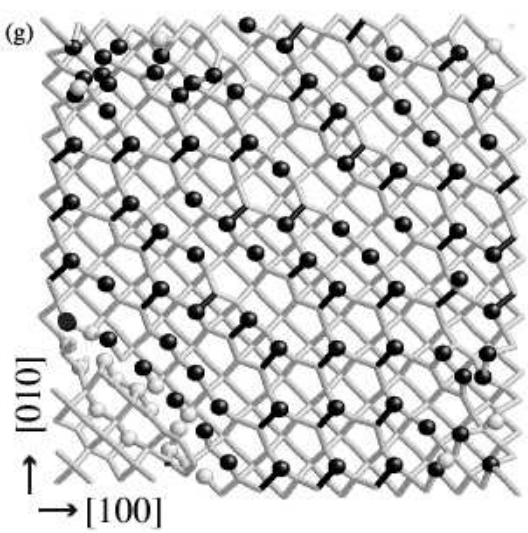

FIG. 3: Snapshots of a fracture process in the (001) plane. The sample size is $n_{100} \times n_{010} \times n_{001}=$ $33 \times 33 \times 33$ (4501 atoms). The time interval between two successive snapshots is $0.3 \mathrm{ps,}$ except that between (f) and (g) (about $1.3 \mathrm{ps).} \mathrm{A} \mathrm{set} \mathrm{of} \mathrm{connected} \mathrm{black} \mathrm{rod} \mathrm{and} \mathrm{black} \mathrm{ball} \mathrm{corresponds}$ to an asymmetric dimer, as in Fig. 2(b) . The left-down area has not yet fractured.

$\varepsilon_{i} \equiv\left\langle\phi_{i}|H| \phi_{i}\right\rangle$ and the hybridization freedom $f_{\mathrm{s}}^{(i)}$ of a Wannier state $\left|\phi_{i}\right\rangle$. Before the bond breaking $(t<0 \mathrm{ps})$, the wave function $\left|\phi_{i}\right\rangle$ is a bonding state in the bulk region, deformed due to the external load. At $t \approx 0 \mathrm{ps}$, a bond breaking occurs and the wave function $\left|\phi_{i}\right\rangle$ loses the bonding character with rapid increase of the bond length. Then ( $0 \mathrm{ps}<t<0.2 \mathrm{ps})$, a twofold coordinated surface atom appears, since another bond is broken almost simultaneously. The wave function $\left|\phi_{i}\right\rangle$ forms a loan pair state that is stabilized by the increase of $f_{\mathrm{s}}^{(i)}(0.6 \rightarrow 0.8)$. The corresponding energy gain can be estimated to be $-0.2 \times\left(\varepsilon_{\mathrm{p}}-\varepsilon_{\mathrm{s}}\right) \approx-1.3 \mathrm{eV}$, which explains the energy gain in the figure $\left(\varepsilon_{i}=-2.7 \mathrm{eV} \rightarrow-3.8 \mathrm{eV}\right)$. In other words, the bond breaking process is caused by the local electronic instability, that is, the energy competition between the loss of the bonding (transfer) energy and the gain due to the increase of the weight on the s orbitals $\left(f_{\mathrm{s}}\right)$. Finally, after the thermal motions with a finite time $(t \approx 0.4 \mathrm{ps})$, a pair of twofold coordinated atoms forms an asymmetric dimer with a $\sigma$ bonding state $\left|\phi_{i}\right\rangle$. The corresponding covalent-bonding energy, defined in Eq. (3) , is $\Delta \varepsilon_{i}^{(\text {cov })} \approx-1.9 \mathrm{eV}$. This energy explains the gain in the figure $\left(\varepsilon_{i}=-3.8 \mathrm{eV} \rightarrow-4.8 \mathrm{eV}\right)$ and the energy loss (about $1.3 \mathrm{eV})$ due to the decrease of $f_{\mathrm{s}}^{(i)}(0.8 \rightarrow 0.6)$. This asymmetric dimer is preserved until the end of the simulation, during a couple of pico seconds. Figure 2(b) is an example of 
observed asymmetric dimers.
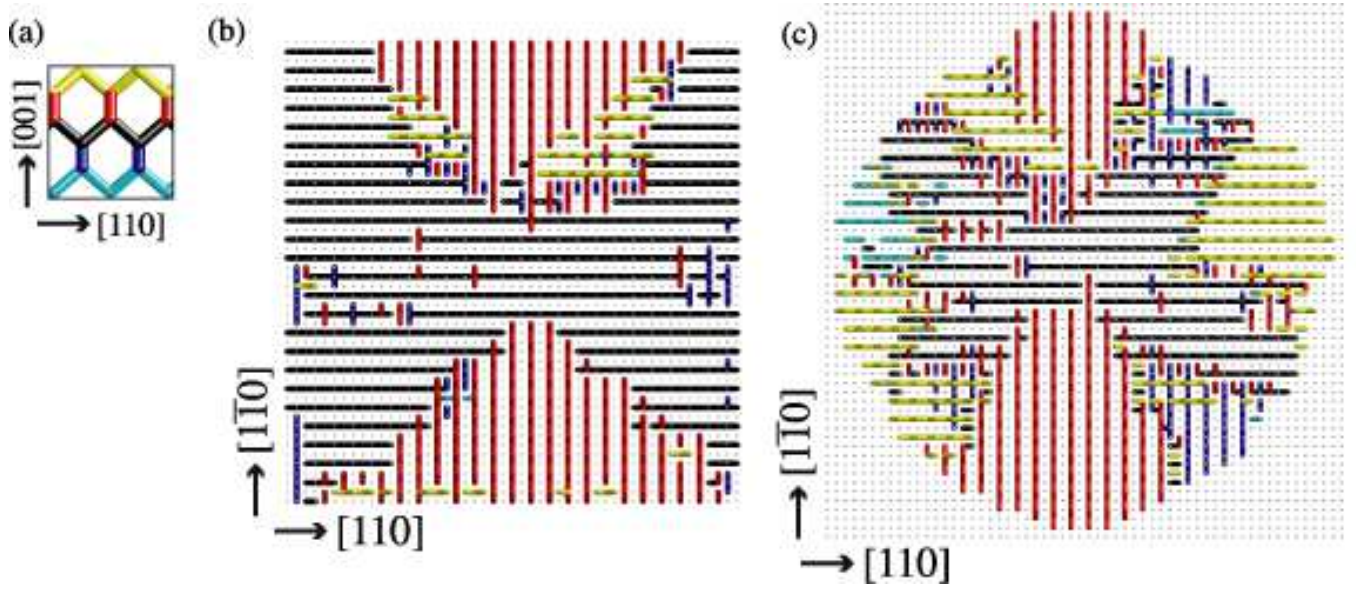

FIG. 4: (a) Ideal diamond structure with colored bond sites. (b)(c) Geometry of resultant cracks in the (001) plane. The broken bond sites are plotted as colored rods in the ideal (crystalline) geometry. Rods within one layer are painted in a same color, as in (a). The layer of black rods contains the defect bond at its central area. Atoms are plotted as dots. The sample sizes in (b) and (c) are $n_{110} \times n_{1 \overline{1} 0} \times n_{001}=49 \times 50 \times 49(30025$ atoms $)$ and $97 \times 100 \times 49(118850$ atoms $)$, respectively. In $(\mathrm{c})$, only a central area $\left(n_{110} \times n_{1 \overline{1} 0}=58 \times 60\right)$ of the sample is shown. Note that the length of $n_{110}=50$ atomic layers is about $10 \mathrm{~nm}$.

Figure 3 shows the fracture process of a cubic sample with 4501 atoms. Each Wannier state is classified from its weight distribution into a bonding or atomic orbital, which is shown as a rod or a ball in the figures, respectively. A black rod or ball corresponds to one in the layer that contains the defect bond. One almost flat (001) surface is being created with many asymmetric dimers. The surface contains, however, many twofold coordinated atoms that have two back bonds (white rods) and a lone pair orbital (black ball). This is because a lone pair states are metastable, as discussed above. In Fig. 3, an anisotropic bond-breaking propagation is seen in the [110] and [1ํㅣ] directions, especially in the early snapshots. In the [110] direction, the successive bond breakings propagate along the nearest neighbor bond sites, which forms a zigzag path, as the black rods in Fig. 4(a). A bond breaking process drastically weakens the nearest neighbor bonds, due to the local electronic instability, observed in Fig. 2(a). Therefore, the successive bond breakings propagate easily in the [110] direction. In the [1ํㅣ direction, on the other hand, the bond-breaking paths 
are not connected, as the red rods in Fig. 4(a). In this direction, the bond breakings are propagated through the local strain relaxation, not by the local electronic instability. As results, the bond breaking propagation along the nearest neighbor bond sites (in the [110] direction of the present surface) is faster than that in the perpendicular direction (in the [110] direction), due to the difference of the successive bond breaking mechanisms. Note that a flat (001) surface is also obtained by a similar simulation without the initial defect bond, in which the fracture begins at the sample edges.

Figures 4 (b) and (c) show larger samples with step formations $\frac{17}{}$. In the two cases, all the conditions are the same, except the sample sizes. To see the step structures clearly, the broken bond sites are shown as rods in the ideal crystalline geometry. The defect bond is located in the center of the drawn area. The anisotropic fracture propagation in one (001) plane increases the anisotropic strain energy $\stackrel{18}{\underline{2}}$. The anisotropy originates from the inequivalence between the [110] and [110] directions within one (001) layer. Since the above inequivalence does not appear within two successive layers, a step formation between them will release the anisotropic strain energy. In Fig. 4 (b), a step is formed between the layer of black rods and that of red rods. In the [110] direction, the bond-breaking propagation reaches the sample surfaces without step formations. In the [1ㅣㅣ directions, the bondbreakings propagate slower and a step is formed in the central area at an early period of the crack propagation. After that, the fracture propagates among the two atomic layers. Since the two layers are symmetrically equivalent, the resultant step formation path, is almost a line in the [100] or [010] directions, as the boundary of the fractured areas between the two layers.

In Fig 4(c), the largest sample in the present letter, the above line structure does not reach the sample surfaces but is canceled with additional step formations in complicated paths. The sample size dependence of the step structures is understood by the beginning of the crossover between nanoscale and macroscale samples; If the sample contains so many atoms, the geometry of the resultant crack will be almost circular, as in Fig. 4. (c), so as to minimize the anisotropic strain energy ${ }^{18}$. If not, the strain energy is accumulated only within the confined bulk region due to the finite sample size. The resultant fracture behavior is directly related to the anisotropic atomic structure of the cleaved surface, as in Fig. (4) (b).

Since the above mechanism of step formations is two-dimensional, the present samples may be nanoscale 'thin' samples. In larger or thicker samples, an expected fracture behavior 
is the bending of the fracture plane into the (111) plane, the easiest cleavage plane in macroscale samples, which is the crossover in the present context. In an enough large sample, the fracture mode with the easiest cleavage plane will grow with no regard for sample shapes and details of conditions. Note that the dynamical simulation with $10^{5}$ atoms is the practical limitation within a single CPU workstation and the program code with parallel computations is now being developed for simulations with larger samples.

This letter shows a possible difference in fracture behaviors among nanoscale and macroscale silicon crystals. Its origin is the size dependence of the energy competition between bulk and surface regions. The electronic structures between the two regions are essentially different and can be described by the present method with the well-defined total energy. This energy competition is also inherent in other phenomena, such as crystal growth and self organizations, which may be candidates for applications of the present method.

This work is supported by a Grant-in-Aid for COE Research 'Spin-Charge-Photon' and a Grant-in-Aid from the Japan Ministry of Education, Science, Sports and Culture. This work is also supported by 'Research and Development for Applying Advanced Computational Science and Technology' of Japan Science and Technology Corporation.

1 A. A. Griffith, Philos. Trans. R. Soc. London, Ser. A 221, 163 (1920).

2 See textbooks of fracture, such as B. Lawn, Fracture of Brittle Solids, 2nd ed., Cambridge University Press (1993).

3 K. C. Pandey, Phys. Rev. Lett. 47, 1913 (1981).

4 F. Ancilotto, et al., Phys. Rev. Lett. 65, 3148 (1990).

5 For example, L. D. Landau and E. M. Lifshitz, Statistical Physics, 3rd ed. Part I, Pergamon Press, Oxford (1980).

6 J. C. H. Spence, Y. M. Huang, and O. Sankey, Acta metall. mater. 41, 2815 (1993).

7 R. Pérez and P. Gumbsch, Phys. Rev. Lett. 84, 5347 (2000).

8 T. Cramer, A. Wanner, and P. Gumbsch, Phys. Rev. Lett. 85, 788 (2000).

9 D. Holland and M. Marder, Phys. Rev. Lett. 80, 746 (1998).

10 T.Hoshi and T.Fujiwara, J. Phys. Soc. Jpn, 69, No.12, 3773 (2000).

11 T.Hoshi and T.Fujiwara, Surf. Sci. 493, 659 (2001). 
12 I. Kwon, et al., Phys. Rev. B 49, 7242 (1994).

13 For example, C.-C. Fu, M. Weissmann, A. Saúl, Surf. Sci. 494,119 (2001).

14 D. J. Chadi, Phys. Rev. Lett. 43, 43 (1979).

15 A Ramstad, G. Brocks, and P. J. Kelly, Phys. Rev. B 51, 14504 (1995).

16 S. Nose, Mol. Phys. 52, 255 (1984).

17 The steps in the (001) surface are categorized in four types (D. J. Chadi, Phys. Rev. Lett. 59, 1691 (1987)). The present surfaces, however, contain unreconstructed domains and are different from the above ones.

18 The elastic property of silicon crystal shows only a small anisotropy within (001) plane; the [110] and [110] directions are equivalent and the values of the Young modulus are different by only about $30 \%$ in the [100] and [110] directions. 